\title{
Multiphasic, Multistructured and Hierarchical Strategies for Cartilage Regeneration
}

\author{
Clara R. Correia, Rui L. Reis, and João F. Mano
}

\begin{abstract}
Cartilage tissue is a complex nonlinear, viscoelastic, anisotropic, and multiphasic material with a very low coefficient of friction, which allows to withstand millions of cycles of joint loading over decades of wear. Upon damage, cartilage tissue has a low self-reparative capacity due to the lack of neural connections, vascularization, and a latent pool of stem/chondroprogenitor cells. Therefore, the healing of articular cartilage defects remains a significant clinical challenge, affecting millions of people worldwide. A plethora of biomaterials have been proposed to fabricate devices for cartilage regeneration, assuming a wide range of forms and structures, such as sponges, hydrogels, capsules, fibers, and microparticles. In common, the fabricated devices were designed taking in consideration that to fully achieve the regeneration of functional cartilage it is mandatory a well-orchestrated interplay of biomechanical properties, unique hierarchical structures, extracellular matrix (ECM), and bioactive factors. In fact, the main challenge in cartilage tissue engineering is to design an engineered device able to mimic the highly organized zonal architecture of articular cartilage, specifically its spatiomechanical properties and ECM composition, while inducing chondrogenesis, either by the proliferation of chondrocytes or by stimulating the chondrogenic differentiation
\end{abstract}

C.R. Correia • R.L. Reis • J.F. Mano, Ph.D. $(\bowtie)$

Department of Polymer Engineering, 3B's Research

Group - Biomaterials, Biodegradables,

and Biomimetics, University of Minho, Headquarters

of the European Institute of Excellence on Tissue

Engineering and Regenerative Medicine, AvePark,

Zona Industrial da Gandra, Barco 4805-017,

Guimarães, Portugal

ICVS/3B's - PT Government Associate Laboratory,

Braga, Guimarães, Portugal

e-mail: jmano@dep.uminho.pt 
of stem/chondro-progenitor cells. In this chapter we present the recent advances in the development of innovative and complex biomaterials that fulfill the required structural key elements for cartilage regeneration. In particular, multiphasic, multiscale, multilayered, and hierarchical strategies composed by single or multiple biomaterials combined in a welldefined structure will be addressed. Those strategies include biomimetic scaffolds mimicking the structure of articular cartilage or engineered scaffolds as models of research to fully understand the biological mechanisms that influence the regeneration of cartilage tissue.

\section{Keywords}

Cartilage regeneration - Hierarchical scaffolds - Multiphasic scaffolds • Chodrogenesis $\bullet$ Stem cells $\bullet$ Tissue engineering

\subsection{Introduction}

Articular (hyaline) cartilage is the thin connective tissue covering the bearing surface of long bones in diarthrodial (synovial) joints, which represent important organs of the musculoskeletal system. An healthy articular cartilage tissue ensures physiological mobility by providing a lubricated and smooth surface, as well as by dissipating energy during the articulation of the joints, which encounter forces of several times the body weight (Mow et al. 1992). The unique composition and highly organized structure of this inhomogeneous, anisotropic, multiphasic, and viscoelastic tissue are the key elements for its complex mechanical properties (Malda et al. 2012). Articular cartilage damage can occur either by degenerative injury or by trauma, which can be acute or repetitive micro trauma from years of use. However, this complex and hierarchical tissue has a very limited capacity of self-repair or regenerate through endogenous healing due to its relatively hypocellular structure, with approximately $100 \times 10^{6}$ chondrocytes $/ \mathrm{cm}^{3}$ on average (Chiang and Jiang 2009), as well as to the lack of neural connections, vascularization, and a latent niche of stem cells/chondroprogenitors (Ge et al. 2012). In current clinical practice, different cartilage repair techniques are being used to address tissue damage. While conservative treatment aims to alleviate pain and slow down or prevent the progress of osteoarthritis (OA), surgical interventions aim to stimulate the natural repair process to rebuild the damaged tissue (Chiang and Jiang 2009; Gomoll and Minas 2014). However, the outcomes of these clinical treatments remain unpredictable and numerous surgical cases have not yet proven to be successful for long-term applications (Chiang and Jiang 2009). Lesions left unrepaired or which have undergone improper repair, subsequently form tissues of inferior mechanical strength, leading to the loss of a suitable mechanical function and progressive OA degeneration, resulting in significant pain and disability.

Cartilage tissue engineering (TE) has emerged as an alternative treatment method of this uniquely challenging tissue. The main challenge to produce functional devices that successfully regenerate cartilage is to mimic its highly organized zonal architecture, specifically its spatiomechanical features and extracellular matrix (ECM) composition. Cartilage TE has employed a variety of techniques to produce scaffolds, frequently composed by multiple components, which are assembled into a final biomimetic three-dimensional (3D) shape. The biomaterials employed include a wide range of synthetic and/ or natural materials, which act as a carrier of and delivery vehicle for, alone or co-cultured, chondrocytes and stem/chondro-progenitor cells towards cartilage formation (Ge et al. 2012).

In this chapter, recent research progress on multistructured strategies to regenerate cartilage is addressed. The reported multistructured strategies 
are divided into four main sections, namely multiphasic, multiscale, multilayered, and hierarchical strategies. Recent progress in this field is summarized and future directions for the development of functional biomaterials are proposed.

\subsection{The Hierarchical Composition of Articular Cartilage}

In the past decade, significant advances have been made in cartilage TE strategies. Most of these efforts have been focused in mimicking its hierarchical and highly organized zonal architecture. In fact, it is well accepted by the scientific community that functional cartilage regeneration can only be achieved through a well-orchestrated interplay of biomechanical properties, unique hierarchical structures, ECM composition, and bioactive factors to stimulate the proliferation of chondrocytes within the engineered biomaterials (Ge et al. 2012). Therefore, to propose successful biomimetic strategies to regenerate cartilage it is required to fully understand, anatomically and functionally, this complex tissue from its macro to nanostructure.

Articular cartilage is a composite and organic solid matrix saturated with water and mobile ions. The stiff and elastic crosslinked collagen fibers present within the ECM of cartilage, predominately type II, provide resistance to shear and tensile forces. Additionally, cartilage is composed of glycoproteins, specifically the proteoglycan aggrecan, that with its highly sulfated glycosaminoglycan (GAG) chain attached to collagen fibers captures large amounts of ions and water molecules via negative charges (Gomoll and Minas 2014). Overall, water constitutes $70-80 \%$ of the wet weight of healthy cartilage, with collagen and to a lesser extent glycoproteins making up the remaining weight. Furthermore, the solid matrix of articular cartilage has a highly hierarchical structural arrangement consisting of four spatially distinct zones, namely (i) the superficial/tangential zone, (ii) the middle/transitional zone, (iii) the deep/radial zone, and (iv) the calcified zone (Poole et al. 2001). Each individual zone is characterized by specific ECM composition and organization, and thus distinct mechanical properties. In the superficial/tangential zone, collagen fibers are oriented parallel to the articular surface and impart high tensile strength to withstand the tensile stress encountered under joint loads, as well as a low coefficient of friction to assure a smooth articulation. This zone has high levels of collagen II and low levels of GAGs. Nevertheless, the relative small amount of proteoglycan molecules in the ECM region contribute to the compressive mechanical properties by producing high osmotic pressure within the tissue (Kleine et al. 2009). In the middle/transitional zone, the collagen fibers are randomly oriented and aggrecan reaches its maximal concentration. Here, the collagen II content decreases, while GAG concentration increases. The deep/radial zone contains the highest concentration of GAGs and the lowest concentration of collagen II fibers. In this zone the collagen fibers are oriented perpendicular and attached to the calcified cartilage zone. Finally, the calcified cartilage zone has high levels of collagen $\mathrm{X}$ and integrates the cartilage to the subchondral bone (Bhosale and Richarson 2008). The mechanical properties of articular cartilage are sensitive to this depth-varying ECM composition. Although the total collagen content per wet weight is unchanged with depth (Malda et al. 2012), hydroxylysine and hydroxylysyl crosslinking increase. This feature combined with the presence of other minor collagen isoforms, such as type IX and XI, play a critical role in regulating collagen fibers diameter, as well as inter-fibers crosslinking, and interactions with cartilage proteoglycans, thereby contributing to the complex mechanical features of the tissue (Bank et al. 1998). Therefore, from the articular surface to the middle/transitional zone, as the concentration of these proteoglycans aggregates increases, the swelling pressure and water content also increase, leading to the ultimately increase of the compressive modulus (Williamson et al. 2003). Besides the depth-varying mechanical features of cartilage, the superficial/tangential, middle/transitional, and deep/radial zones have distinct cell morphology and metabolic activity, with distinct 
Scheme 9.1 Illustration of articular cartilage tissue. From the superficial/tangential to the deep/radial zones of articular cartilage the increasing trend of water, proteoglycans, and collagen II contents, and collagen fibers diameter are represented. On the opposite direction, the increasing trend of glycosaminoglycans and collagen $\mathrm{X}$ content, and compressive modulus values are also illustrated

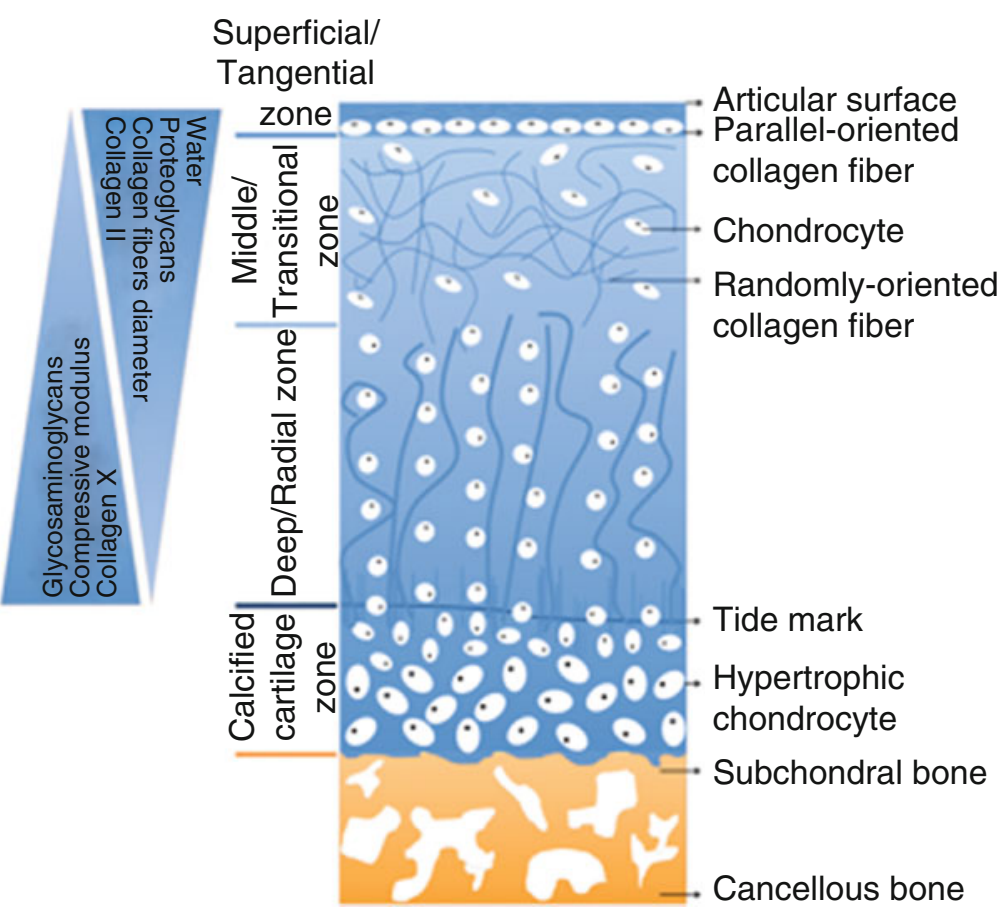

gene and microRNA expression profiles (Grogan et al. 2013). Chondrocytes in the superficial/tangential zone are flattened and horizontally clustered at a relatively high density, in the middle/ transitional they are more rounded and randomly oriented in the ECM, and in the deep/radial zone chondrocytes are larger and organized in vertical columns. Scheme 9.1 summarizes the described spatially-varying native articular cartilage anatomy. From the superficial/tangential to the deep/ radial zones of articular cartilage, the decreasing trend of water, proteoglycans and collagen II content, as well as fibers diameter are represented. On the opposite direction, the decreasing trend of GAGs and collagen X content, as well as compressive modulus values are also illustrated.

\subsection{Research Progress on Cartilage Regeneration Strategies}

Among the different employed biomaterials to produce scaffolds for cartilage TE, naturally occurring polymers, such as collagen and polysaccharides, have been widely used since they resemble the natural ECM composition of cartilage, rich in GAGs. Examples of natural biomaterials frequently used in cartilage $\mathrm{TE}$ strategies are collagen I and II, alginate, agarose, chondroitin sulfate, chitosan, hyaluronic acid, fibrin, among others, as summarized in the literature (Chung and Burdick 2008). Although natural biomaterials are recognized and interact with cells via surface receptors, consequently regulating or directing their biological behavior and phenotype, they also stimulate an immune system response of the body upon implantation. Therefore, antigenicity and disease transfer are often a concern when scaffolds composed by natural polymers are implanted. Additionally, natural biomaterials are subject to variable enzymatic host degradation and thus have a fast degradation rate. To address those issues, they are often combined with synthetic polymers, which are more controllable and predictable, since their chemical and physical properties can be modified to alter mechanical and degradation characteristics. A wide range of synthetic polymers have been used for different biomedical applications as summarized in the literature (Tian et al. 2011). While natural biomaterials provide the biological cues to promote cell adhesion and proliferation, and mimic the natural ECM 
composition of cartilage, synthetic polymers allow mimicking the adjacent complex mechanical cues. Different combinations of natural biomaterials and synthetic polymers to produce scaffolds for cartilage tissue engineering are reported in the literature. For example, chitosan was grafted to poly( $\varepsilon$-caprolactone) (CH-PCL) to produce grafting copolymers with a tailored mechanical and degradation properties (Wan et al. 2010). Recently, CH-PCL grafting copolymers were blended with collagen II to fabricate stratified and multilayered porous scaffolds for cartilage TE (Zhu et al. 2014). In fact, the production of an engineered cartilage tissue has encountered specific difficulties because articular cartilage has a stratified structure, with an ECM composition and mechanical properties varying in an apparently anisotropic fashion. The classical TE approach of creating homogeneous tissue replacements for articular cartilage has failed to achieve widespread clinical effectiveness because the bulk properties of the homogeneous tissue substitutes do not mimic native tissue function. Considering that the properties of cartilage are dictated by its spatiallyvarying structural organization, current $\mathrm{TE}$ approaches are focused in reproducing the native architecture and function of cartilage. Therefore, the manufacture of multistructured and hierarchical scaffolds with satisfactory structures and properties that mimic those found in the native tissue is now a hot topic in cartilage TE and regenerative medicine.

In this section we discuss different strategies reported in the literature to produce multistructured and hierarchical 3D structures for cartilage regeneration. Depending on the concept adopted, those strategies are divided in four sections, namely (i) multiphasic, (ii) multiscale, (iii) multilayered, and (iv) hierarchical strategies. Mainly, these strategies aim to create 3D constructs with variable molecular compositions and mechanical properties, mimicking the heterogeneous ECM composition and consequently zone-variable mechanical properties of native cartilage. Additionally, strategies to produce multistructured or hierarchical scaffolds as research models to fully understand the biological cues that dictate the successful regeneration of cartilage were also included.

\subsubsection{Multiphasic Strategies}

Hydrogels are widely used in cartilage TE due to their inherent biocompatibility, high water content, and resemble to the natural cartilage ECM. However, hydrogels have limited mechanical strength, which is a main issue for cartilage regeneration. The recreation of the complex mechanical properties of native healthy cartilage is of great importance, especially since upon implantation the construct must provide ability to withstand physiologic joint loading. Alternative strategies have been explored to increase the strength of hydrogel constructs, e.g. through the creation of interpenetrating networks (IPN), incorporation of solid particles, fibers and/or tubes. This lead to the rise of new strategies for cartilage regeneration comprising the combination of biomaterials in different states, namely soft and rigid biomaterials, assembled in a single 3D structure, here termed as multiphasic strategies.

An example of a multiphasic strategy in cartilage TE is the composite 3D woven PCL scaffolds with interpenetrating network (IPN) hydrogels proposed by Liao et al. (2013). A 3D scaffold that combined two material states, namely IPN hydrogels and rigid fibers, was developed. Commonly used hydrogels for cartilage regeneration, such as alginate, agarose, gelatin (Awad et al. 2004), hyaluronic acid or poly(ethylene glycol) (PEG) (Chung et al. 2009), generally consist of polymers of single networks and exhibit relatively low stiffness and wear properties. To address the harsh biomechanical requirements of the joint, IPN hydrogels in different combinations of alginate, fibrin, and polyacrylamide (PAAm) were combined with 3D woven PCL scaffolds. The proposed IPN hydrogels showed a significant improvement in the Young's aggregate and dynamic moduli, as well as low coefficients of friction similar to native cartilage. Additionally, the combination with 3D PCL woven scaffolds ensured compressive properties similar to those found in the native tissue, thus indicating the successful resistance to joint loading after implantation procedures. In the same concept, other examples of multiphasic cartilage TE strategies with improved mechanical 
properties are the co-electrospinning of soft and rigid fibers composed by gelatin and poly(Llactic acid) (PLLA) (Torricelli et al. 2014), respectively, or gelatin methacrylamide (gelMA) hydrogels reinforced with $3 \mathrm{D}$ printed scaffolds composed by a thermoplastic polymer blend between poly(hydroxymethylglycolide-co- $\varepsilon$ caprolactone) (pHMGCL) and PCL. pHMGCL/ PCL was also functionalized with methacrylate groups (pMHMGCL). The 3D composite scaffold with and without methacrylate groups (Ma+ and MA-, respectively) were tested in a PLLA model of the femoral condyle of the human knee for a focal articular cartilage defect (Boere et al. 2014).

Multiphasic cartilage TE strategies can also comprise controlled drug release strategies that take advantage of the combination of different material states in the same 3D construct. Spiller et al. (2012) developed a complex multiphasic strategy to regenerate cartilage taking in consideration the importance of cartilage formation and integration, the sustained release of cartilage growth factors, and the required mechanical properties of the engineered device to successfully regenerate cartilage. For that, they produced different building blocks, namely a poly(glycolic acid) (PGA) fiber mesh scaffold, a poly(vinyl alcohol) (PVA) hydrogel, and poly(lactic-coglycolic acid) (PLGA) microparticles loaded with insulin-like growth factor-1(IGF-1). The PLGA microparticles loaded with IGF-1 were then encapsulated in the PVA hydrogel, and the whole 3D construct was finally wrapped with PGA fibers. In vivo results showed that the outer layer composed by PGA fibers improved cartilage formation and integration, while the inner core composed by PVA/PLGA hydrogel provided mechanical support while releasing IGF-1 in a sustained fashion.

Figure 9.1 summarizes the referred examples found in literature comprising multiphasic strategies to regenerate cartilage.

\subsubsection{Multiscale Strategies}

Collagen, proteoglycans, noncollagenous proteins, and tissue fluid compose the ECM of artic- ular cartilage. In particular, collagen II, VI, IX, and XI, are found in articular cartilage, although 90-95\% of total collagen is composed of type II. Collagen types II, IX and XI form fibrils and create an interweaved fiber mesh in the ECM environment, where fibril diameter increases with depth (Temenoff and Mikos 2000). Aiming to mimic this multiscale organization of articular cartilage, different strategies have been proposed, here termed as multiscale strategies.

Fibrous scaffolds are widely used in TE due to their characteristic high porosity and interconnected pores that facilitate cellular infiltration. Previous reports have indicated that the size scale of fiber scaffolds influence tissue development. Specifically, nanoscale features are desirable because resemble better the collagen fibers of the native ECM (Li et al. 2005). However, scaffolds only composed by nanofibers have a limited cellular infiltration due to a closed pore network mesh (Pham et al. 2006). Therefore, the logical progression was the development of scaffolds combining fibers of variable sizes. Levorson et al. (2014) developed electrospun scaffolds with fibers of two diameter scales interspersed evenly throughout an entire 3D construct. For that, nano and micro fibers of PCL, or nano and micro fibers of fibrin and PCL, respectively, were produced. Results showed that the presence of both nanofibers, either synthetic (PCL) or natural (fibrin), within a PCL microfiber mesh improved the proliferation of cells in serum-free conditions, as well as aided the deposition of GAGs compared with scaffolds composed solely of microfibers. In a similar multiscale cartilage strategy, Moroni et al. (2008) combined rapid prototyping fabrication technique of 3D fiber deposition (3DF) with electrospinning (ESP) to fabricate integrated macro and micro polymeric scaffolds. Scaffolds consisted of integrated 3DF periodical macrofiber and random ESP microfiber networks (3DFESP) were produced with block-co-polymers of poly(ethylene oxide terephthalate) (PEOT) and poly(butylene terephthalate). While 3DF macro scaffolds provided structural integrity and enhanced mechanical properties, the ESP micro network allowed cell entrapment and resembled the nano features of native cartilage ECM. Results showed that 

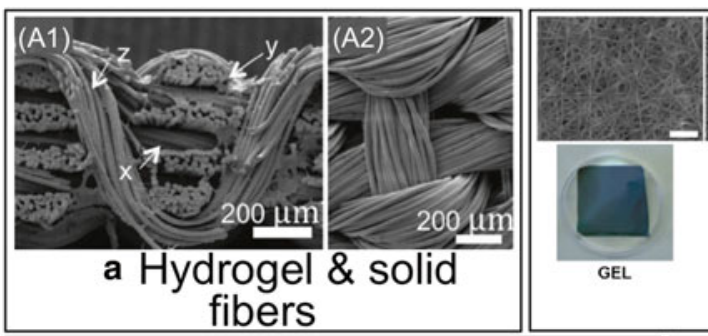

GEL

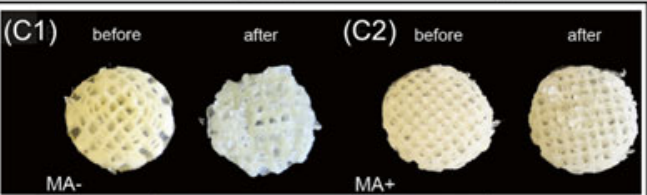

$(\mathrm{C} 3)$

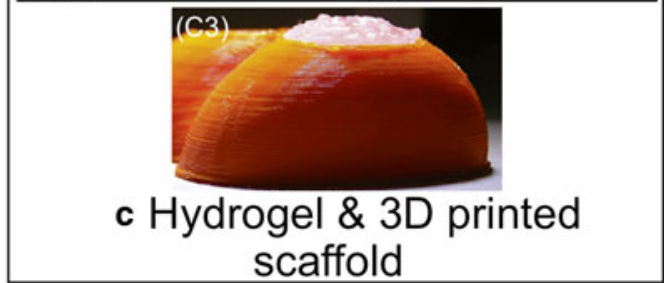
scaffold

Fig.9.1 Examples of multiphasic strategies to regenerate articular cartilage. (a) 3D woven PCL/IPN composite composed by (Al) stacked layers, namely seven layers of $x$ and $y$ fiber bundles of PCL, which were interlocked with a third set of PCL fibers ( $z$-direction) that were passed vertically through the layers following a continuous and repeated path as showed in (A2) (Adapted from Liao et al. 2013). (b) Scanning electron microscopy images and photographs of crosslinked mats composed by co-electrospun fibers of gelatin (GEL) and PLLA. Scaffolds containing different amounts of PLLA and gelatin (nominal PLLA/ gelatin weight ratio: 0/100, 30/70, 50/50, 70/30, 100/0) were produced. Scale bar is $10 \mu \mathrm{m}$ (Adapted from Torricelli et al. 2014). (c) Gelatin methacrylamide hydrogels reinforced with a thermoplastic polymer blend of

3DFESP scaffolds had a superior cell entrapment, GAG/DNA ratio, and expression of sulfated GAGs by safranin-O staining compared to 3DF scaffolds. Other groups besides varying the fiber diameter also tested the orientation of the fibers. McCullen et al. (2012) reported the fabrication of a trilaminar scaffold by sequential electrospinning and varying fiber size and orientation in a continuous construct, to create scaffolds that mimicked the structural organization and mechanical properties of the collagen fiber network of cartilage. By preparing PCL solutions with different concentrations and by varying the linear velocity of the collector, fibers with variable diameters (1 and $5 \mu \mathrm{m}$ ) and aligned or ran-

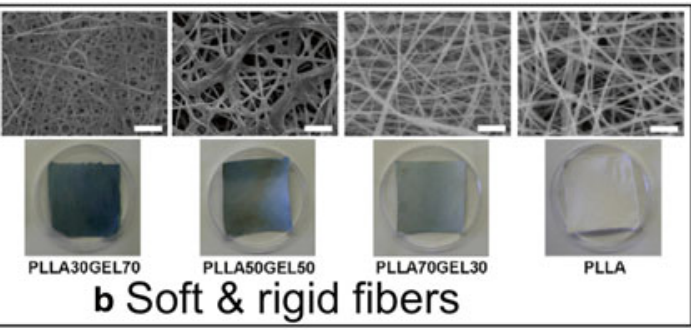

b Soft \& rigid fibers

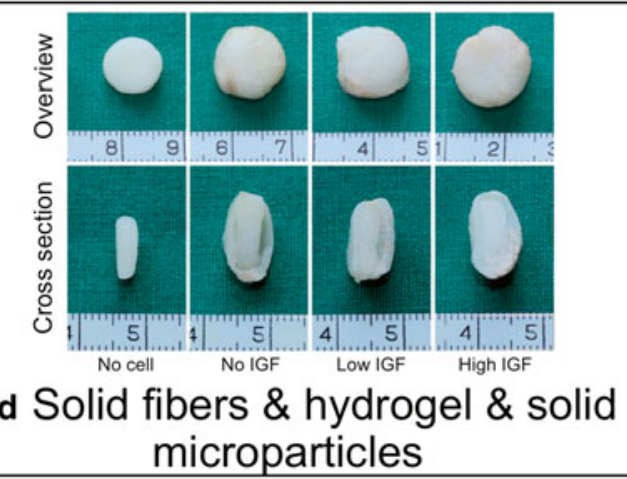

poly(hydroxymethylglycolide-co- $\varepsilon$-caprolactone)/PCL functionalized with methacrylate groups $(\mathrm{MA}+)$ or without (MA-). Scaffolds were photographed $(C 1)$ before and (C2) after applying increasing axial compressive forces. (C3) The 3D composite scaffolds were tested in a model (side view) of the femoral condyle of the human knee composed by PLLA (orange) (Adapted from Boere et al. 2014). (d) PGA fiber mesh scaffold wrapping a PVA hydrogel encapsulating PLGA microparticles loaded with IGF-1. Different formulations of composite scaffolds were tested namely a control without cells (No cell), with unloaded microparticles (No IGF), with a low concentration of IGF-1 (Low IGF), and with a high concentration of IGF (High IGF) (Adapted from Spiller et al. 2012)

domly oriented were produced. Three different zones aiming to mimic the superficial, middle, and deep natural zones of cartilage composed the developed trilaminar construct. Results demonstrated that the trilaminar scaffold displayed superior mechanical properties compared to homogeneous scaffolds, and supported in vitro cartilage formation.

Besides exploring the fibers diameter from a macro to a nanoscale, other groups included carbon nanotubes to regenerate cartilage. Carbon nanotubes mimic the dimensions of the constituent components of tissues, in which cells interact with nanofibrous proteins. This feature makes carbon nanotubes excellent candidates when 
employed as implants (Tran et al. 2009). In particular, carbon nanotubes can be used as a secondary phase for high load bearing such as cartilage applications, due to their superior mechanical properties. Additionally, the unique chemical properties of carbon nanotubes allows them to be functionalized with different chemical groups to, for example, improve cell adhesion and proliferation. Holmes et al. (2013) combined wet and electrospinning to fabricate poly(L-lactic acid) (PLLA) fiber scaffolds with a controlled fiber dimension and combined with multi-walled carbon nanotubes (MWCNTs). The MWCNTs used were treated or untreated with hydrogen $\left(\mathrm{H}_{2}\right)$. In vitro studies with mesenchymal stem cells (MSCs) showed that PLLA scaffolds composed of fibers with smaller diameters had improved cell adhesion. More importantly, the incorporation of MWCNTs in the PLLA fiber scaffolds had a drastic increase in mechanical strength and a compressive modulus similar to native cartilage. Ultimately, the chondrogenic differentiation of MSCs was enhanced by combining $\mathrm{H}_{2}$ MWCNTs-PLLA fiber scaffolds with poly(L-lysine) (PLL) coating, resulting in the highest GAGs content compared to the control scaffolds, namely PLLA, MWCNTsPLLA, and $\mathrm{H}_{2}$ MWCNTs-PLLA.

Another type of a multiscale strategy to regenerate cartilage is to create in the same structure a gradient pore size as reported by Zhang et al. (2013). A collagen porous scaffold by using ice particulates as the porogen material with four ranges, namely $150-250,250-355,355-425$, and 425-500 $\mu \mathrm{m}$, within the same construct was developed. The objective of the study was not to mimic the multistructured organization of cartilage, but to create a research model to study in the same construct, and thus under the same culture experiment conditions, the effect of pore size on cartilaginous matrix production and cartilage regeneration.
Multiscale strategies also include the encapsulation of smaller structures within macrostructures, which is a frequently employed cartilage TE strategy, particularly in drug delivery systems. The most common approach is the encapsulation of hydrogel microparticles within a macro hydrogel structure. Bian et al. (2011) reported a multiscale delivery system able to support human MSCs chondrogenesis and neocartilage formation. The system was composed of alginate microparticles coated with transforming growth factor- $\beta 3$ (TGF- $\beta 3$ ) nanofilm and encapsulated within a hyaluronic acid (HA) hydrogel. The sustained release of TGF- $\beta 3$ from microparticles resulted in an enhanced expression of chondrogenic markers and higher levels of cartilage specific matrix deposition by human MSCs in HA hydrogels compared to uncoated microspheres (negative control) and, more importantly, with HA hydrogels without microparticles and, thus, with TGF- $\beta 3$ directly encapsulated within the matrix core of the hydrogel. Additionally, the in vivo results showed that the sustained release of TGF- $\beta 3$ provided by the multiscale strategy was essential to neocartilage formation compared to the burst release of TGF- $\beta 3$ directly loaded in the core of HA hydrogels. In the same concept, Nanda et al. (2014) reported a strategy of a porous scaffold encapsulating microparticles for cartilage regeneration. A freeze-dried collagen porous scaffold with a controlled porous structure and incorporating insulin loaded PLGA microparticles was developed. The proposed hybrid scaffold demonstrated a high mechanical strength and a sustained release of insulin for 4 weeks, ensuring the in vitro survival and proliferation of chondrocytes.

Figure 9.2 summarizes the referred examples found in literature comprising multiscale strategies to regenerate cartilage.
Fig.9.2 (continued) cells (MSCs) and alginate microparticles with a TGF- $\beta 3$ nanofilm. (F1) Schematic representation of the photoencapsulation (UV light) to produce loaded MeHA hydrogels. (F2) Fabricated HA hydrogel disk and fluorescent and bright field microscopic images of MSCs (membrane labeled with red dye) and alginate microspheres (containing FITC-labeled protein) encapsulated in HA gels (Adapted from Bian et al. 2011). (g) Scanning electron microscopy images of $(G 1)$ freezedried collagen porous scaffolds (control) and (G2) scaffolds incorporating insulin loaded PLGA microparticles. $(G 3)$ and (G4) are high magnification images of $(G 1)$ and $(G 2)$, respectively. The yellow arrows at $(G 4)$ represent the integrated PLGA microparticles in the porous collagen matrix (Adapted from Nanda et al. 2014) 

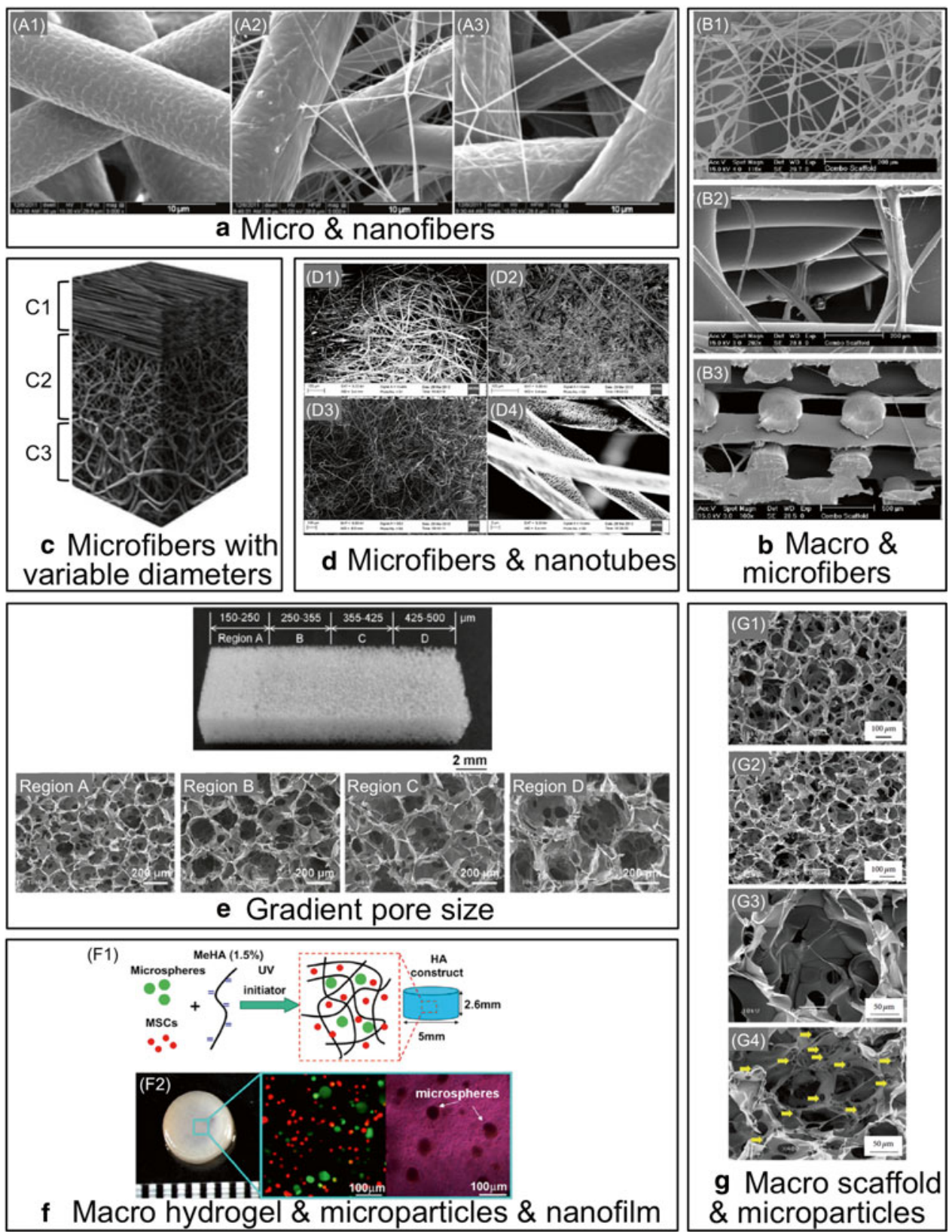

Fig. 9.2 Examples of multiscale strategies to regenerate articular cartilage. (a) Electrospun scaffolds with fibers of two diameter scales interspersed evenly throughout an entire 3D construct. (A1) PCL microfibers, (A2) PCL micro and nanofibers, and (A3) PCL microfibers and fibrin nanofibers (Adapted from Levorson et al. 2014). (b) Macro scaffolds of integrated 3D fiber ( $3 D F$ ) deposited by rapid prototyping technique and electrospun $(E S P)$ microfibers with block-co-polymers of poly(ethylene oxide terephthalate) (PEOT) and poly(butylene terephthalate). ESP network spun for (B1) 2 min or (B2) $30 \mathrm{~s}$ every two 3DF deposited layers. Scale bar is $200 \mu \mathrm{m}$. (B3) Cross section of (B2). Scale bar is $500 \mu \mathrm{m}$ (Adapted from Moroni et al. 2008). (c) Trilaminar scaffold produced by sequential electrospinning. The $3 \mathrm{D}$ construct was composed by three different zones by varying the diameter and organization of PCL microfibers: $(C 1)$ superficial zone with aligned $1 \mu \mathrm{m}$ fibers, $(C 2)$ middle zone with random $1 \mu \mathrm{m}$ fibers, and (C3) deep zone with random $5 \mu \mathrm{m}$ fibers (Adapted from McCullen et al. 2012). (d) Scanning electron microscopy images of varying PLLA electrospun fibrous scaffolds with $(D 1) 0.5 \%$ multi-walled carbon nanotubes (MWCNTs), treated with $(D 1) 0.5 \%$ or $(D 2)$ $1 \%$ of hydrogen. (D4) High magnification picture of $(D 1)$ (Adapted from Holmes et al. 2013). (e) Collagen porous scaffolds with gradient pore sizes by using ice particulates as a porogen material. The ice particulates had diameters of $150-250,250-355,355-425$ and $425-500 \mu \mathrm{m}$, originating four different regions through the 3D scaffold, namely region $\mathrm{A}, \mathrm{B}, \mathrm{C}$, and $\mathrm{D}$, respectively (Adapted from Zhang et al. 2013). (f) Methacrylated hyaluronic acid (MeHA) hydrogels encapsulating mesenchymal stem 


\subsubsection{Multilayered Strategies}

Inspired by the depth-dependent multi-zone morphology of articular cartilage,with varied mechanical properties and function, scaffolds composed by organized layers were developed. The most common approach is to vary the content of a biomaterial and create a multilayered 3D scaffold, either in porous scaffolds or hydrogels. Zhu et al. (2014) proposed a porous scaffold composed by collagen II blended to CH-PCL copolymers. By varying the content of collagen II and chitosan in opposite trends, a four-layered porous scaffold similar to the ECM of articular cartilage in terms of composition, porous architecture, water content, and compressive mechanical properties, was developed. Nguyen et al. (2011) developed a three-layer poly(ethylene glycol) (PEG)-based hydrogel with chondroitin sulfate (CS) and matrix metalloproteinasesensitive peptides (MMP-pep) in the top layer, CS in the middle layer, and HA in the bottom layer. Mouse MSCs were encapsulated within the multilayered hydrogels and their biological outcome was assessed. Results demonstrated that the developed hydrogels not only induced the MSCs to differentiate into the chondrogenic lineage but also that the phenotype and matrix production profile could be tailored to specific zones of articular cartilage by altering the material composition alone. Most importantly, the authors showed that the spatial organization of specific biomaterials affected collagen II expression and created a composite tissue structure with spatially-varying collagen II levels, increasing from the superficial layer to the deep layer. Creating layers by varying the shape of the biomaterial used is also an example of a multilayered strategy. Steele et al. (2014) developed a PCL two-zone approach with electrospun fiber articulating surface deposited onto a particulatedleached foam. The final two-layered 3D scaffold was composed by an aligned fiber zone to mimic the morphology of the superficial zone of articular cartilage, laminated to a bulk porous particulate-template scaffold to allow cellular infiltration and extensive ECM deposition. Results showed that the incorporation of aligned fibers significantly enhanced the tensile mechanics of the laminated porous scaffold. Additionally, the fibers also reduced the surface roughness of the porous scaffolds, mimicking the smooth articular surface of native cartilage. On the other hand, the porous scaffolds contributed to the infiltration of seeded chondrocytes, resulting in high rates of proliferation and GAG production. $\mathrm{Ng}$ et al. (2009) proposed a layered agarose hydrogel by varying the population of encapsulate chondrocytes. Chondrocytes from the superficial (SZC) and middle (MZC) zones were isolated and encapsulated to form bilayered constructs with 2 or $3 \%$ agarose. Two different layered formulations were tested, namely $2 \% \mathrm{SZC} / 2$ $\% \mathrm{MZC}$ and $3 \% \mathrm{SZC} / 2 \% \mathrm{MZC}$. For SZC, GAG and collagen production increased with increased agarose concentration and when layered with MZC. For MZC, GAG production and cell proliferation increased when layered with SZC.

Another type of multilayered strategies is the creation of gradients by the incorporation of biomolecules found in the ECM, such as proteins and GAGs, which are known to organize cytokines, chemokines, and growth factors, thus guiding cell migration, growth, and differentiation in various biological processes. However, controlling the spatial distribution of these biomolecules to mimic native tissue remains very challenging. Chow et al. (2014) proposed the incorporation of a peptide-polymer conjugate system to functionalize the surface of scaffolds with selected peptides that specifically and dynamically bind GAGs to guide their spatial arrangement. Combining this functionalization approach with electrospinning, a single and dual opposing gradients of peptide concentrations that directed the spatial organization of GAGs through the thickness of the scaffold was developed. Two binding peptides, namely HA-binding and chondroitin sulfate (CS)-binding peptides-PCL conjugate, were incorporated by co-electrospinning into PCL fibers, producing HAbind-PCL and CSbind-PCL fibers, respectively. Sequential electrospinning of opposing concentrations of HAbind-PCL and CSbind-PCL created peptide gradients, that specifically organized contrasting gradients of HA and CS through the scaffold 
thickness. Interestingly, the gradient in peptide concentrations organized GAGs into a depth gradient through the scaffold thickness. Gradients of biomolecules can also be simply achieved by the encapsulation of varying concentrations within a hydrogel. Smith Callahan et al. (2013) developed a poly(ethylene glycol dimethacrylate) (PEGDM) hydrogel system with a gradient of arginineglycine-aspartic acid peptide (RGD) concentrations to correlate primary human osteoarthritic chondrocyte proliferation, phenotype maintenance, and ECM production. In higher RGD concentration regions, the cell number and the expression of chondrogenic phenotype markers decreased, while, on the other hand, in lower RGD concentration regions both were maintained. Additionally, ECM content was higher in lower RGD concentration regions. The design of gradients through 3D structures was also explored by Thorpe et al. (2013), by modulating the oxygen tension and mechanical environment thorough the depth of MSCs seeded agarose hydrogels. The 3D constructs were radially confined of half their thickness and subjected to dynamic compression (DC). Results showed that confinement led to low oxygen levels in the bottom of the construct, while the application of DC increased strain at the top. These spatial changes correlated with GAGs accumulation in the bottom of the construct and led to increased collagen accumulation in the top, as well as a suppression of hypertrophy and calcification throughout the construct. Therefore, by modulating gradients of the environment through the depth of agarose hydrogels, it was possible to suppress MSCs chondral progression and to engineer tissues with zonal gradients mimicking certain aspects of native articular cartilage.

Figure 9.3 summarizes the referred examples found in literature comprising multilayered strategies to regenerate cartilage.

\subsubsection{Hierarchical Strategies}

Hierarchical strategies to regenerate cartilage comprise organized bottom-up approaches, in which smaller components are assembled into greater 3D constructs in an attempt to mimic the native hierarchical organization of cartilage, from its macro- to nano-stratified cues. For this propose, different assembling techniques have been explored, such as layer-by-layer (LbL) and peptide self-assembly, inverted crystal colloidal (ICC), cell sheets, and magnetic force-based approaches.

The most common approach in hierarchical strategies for cartilage TE is to assembly microparticles and create microparticles-based 3D scaffolds. For example, Silva et al. (2013) developed nanostructured 3D constructs combining LbL technology and template leaching as scaffolds for cartilage regeneration. Paraffin spheres were previously modified with poly(ethyleneimine) (PEI) and used as templates to produce the LbL membrane composed by chitosan and chondroitin sulphate as polyelectrolytes. Once the LbL membrane was built, the paraffin was leached out and samples were freeze-dried. The obtained 3D constructs had high porosity and water uptake, and a viscoelastic nature. Cultures of bovine chondrocytes $(\mathrm{BCH})$ and MSCs were performed up to 21 days. Both cells adhered and proliferated on the surface of the samples. Additionally, during the time of the culture tested, $\mathrm{BCH}$ maintained their chondrogenic phenotype and MSC were able to differentiate into the chondrogenic lineage upon chondrogenic medium stimulation. Singh et al. (2010) explored another assembly approach to produce microparticles-based scaffolds by using subcritical carbon dioxide $\left(\mathrm{CO}_{2}\right)$ as a sintering agent to assemble poly(D,L-lactide-co-glycolide) (PLG) microparticles. The $\mathrm{CO}_{2}$ sintering technique for manufacturing microparticlesbased scaffolds is suitable for producing cellcontaining, shape-specific matrices, such as scaffolds, under relatively mild conditions via a single-step sintering of microparticles in the presence of cells, with high cell viability. In vitro assays using chondrocytes and human umbilical cord mesenchymal stromal cells (HUCMSCs) demonstrated the viability of the method and, more importantly, that the macro constructs were suitable for cartilage tissue engineering applications. 


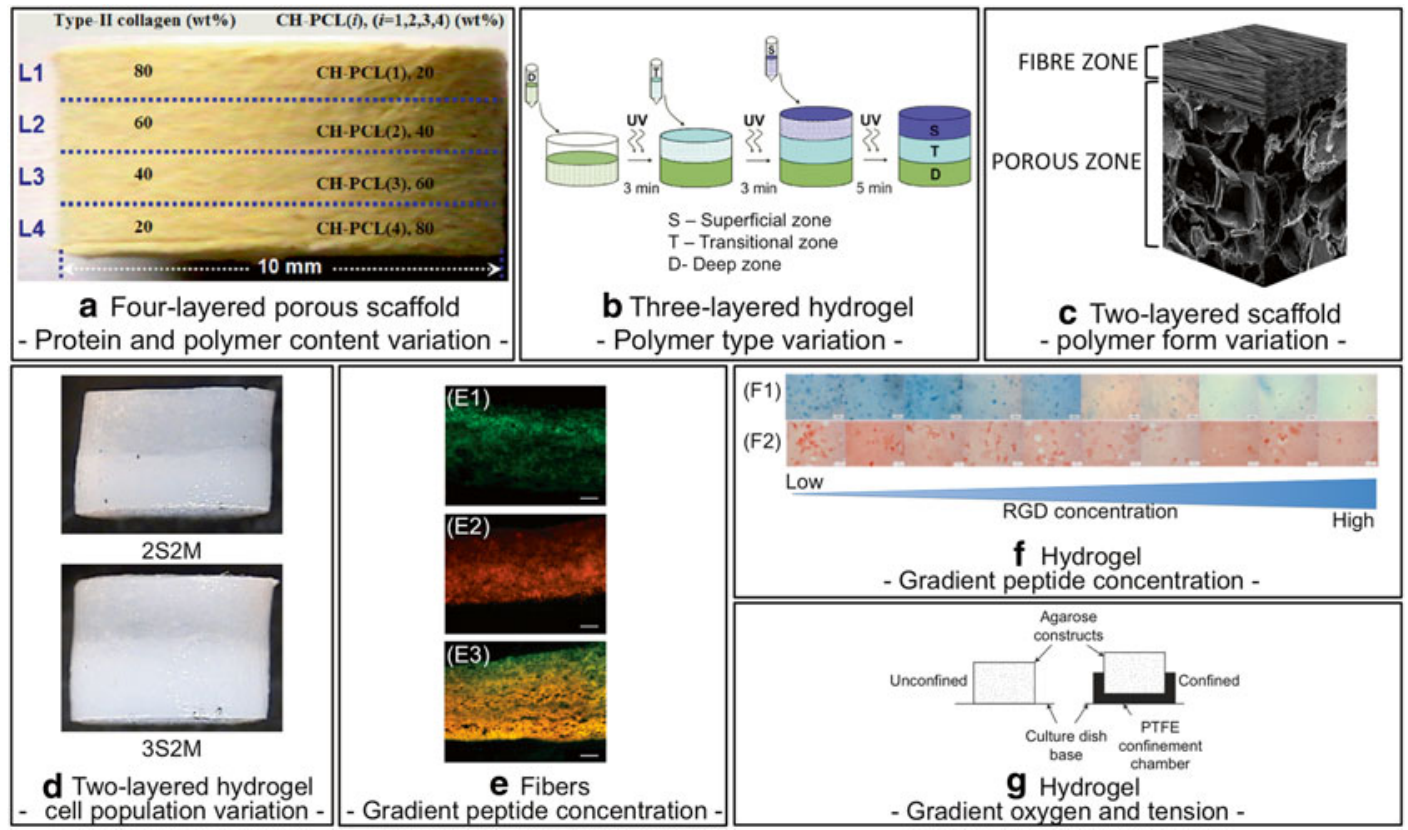

Fig. 9.3 Examples of multilayered strategies to regenerate articular cartilage. (a) Image of a cylindrical collagen II/chitosan-PCL scaffold with the schematic representation of its four layers (L1, L2, L3, and L4), which were achieved by varying the content of collagen II and chitosan in opposite trends. The dotted lines indicate the interface zones of the layers (Adapted from Zhu et al. 2014). (b) Multilayered hydrogel construct fabrication with three distinctive layers, each corresponding to the superficial $(S)$, transitional $(T)$, and deep $(D)$ zones of articular cartilage (Adapted from Nguyen et al. 2011). (c) Diagram illustrating the two-layered PCL scaffold composed by an electrospun PCL fiber zone laminated to a bulk porous particulate-template PCL scaffold (porous zone) (Adapted from Steele et al. 2014). (d) Chondrocytes from the superficial (SZC) and middle (MZC) zones were isolated and encapsulated to form two-layered hydrogels with 2 or $3 \%$ agarose. Two different layered formulations were tested, namely $2 \% \mathrm{SZC} / 2 \% \mathrm{MZC}(2 \mathrm{~S} 2 \mathrm{M})$ and $3 \% \mathrm{SZC} / 2 \% \mathrm{MZC}$ (3S2M) (Adapted from $\mathrm{Ng}$ et al. 2009). (e) Fluorescence microscopy of cross sections of gradient scaffolds formed

Other methodology used to assemble microparticles in hierarchical cartilage TE applications is the production of ICC scaffolds. Organized colloids with hexagonal structure are often described as photonic crystals, photonic band gap materials, and artificial opals since close-packed particles display unique optical properties via the diffraction of visible light. By organizing arrays of microparticles as an ordered reverse replica, a 3D scaffold with inverted lattice by sequential electrospinning concentrations of peptidePCL conjugates of (E1) HAbind-PCL labeled with fluorHA (green), (E2) CSbind-PCL labeled with rhod-CS (red), and (E3) opposing concentrations of HAbind-PCL and CSbind-PCL labeled with fluor-HA and rhod-CS (Adapted from Chow et al. 2014). (f) Poly(ethylene glycol dimethacrylate) (PEGDM) hydrogel system with a gradient of arginine-glycine-aspartic acid peptide $(R G D)$ concentrations. (F1) and (F2) are histological sections of alcian blue and sirius red stainings, respectively. Images of the extracellular matrix production by human chondrocytes after 3 weeks of culture where taken every $5 \mathrm{~mm}$ down the length of the gradient. Scale bar is $200 \mu \mathrm{m}$ (Adapted from Smith Callahan et al. 2013). (g) Experimental design of agarose hydrogel constructs press-fitted into custom made PTFE wells. The 3D constructs were radially confined of half their thickness and subjected to dynamic compression, creating a gradient of oxygen tension and mechanical environment thorough the depth of the hydrogels (Adapted from Thorpe et al. 2013)

pore topography was produced, named ICC scaffolds. Kuo and Tsai (2011) proposed the development of heparinized ICC scaffolds to generate uniform tissue-engineered cartilage. Highly viscous heparin, chitin, and $\mathrm{CH}$ gels were infiltrated repeatedly into the voids among self-assembled polystyrene (PS) microparticles. In vitro assays with chondrocytes showed that cells proliferated, secreted GAGs, and produced collagen. Importantly, the infused cells could migrate and 
proliferate in and near the core of the ICC scaffolds. This uniform cell growth was mainly because the regular pore geometry of ICC scaffolds was able to reduce the resistance to cell migration and nutrient transport. Moreover, immunocytochemistry for collagen II also showed a uniform distribution of cartilaginous components.

In a different approach from microparticlesbased scaffolds, Liu et al. (2011) explored the assembly of nanofibers to produce hollow microparticles without using any prefabricated template. Poly(amidoamine) (PAMAM) dendrimers were used as initiators to synthesize star-shaped PLLA (SS-PLLA), which self-assembled into nanofibrous hollow microparticles. The engineered microparticles were evaluated as injectable cell carriers for cartilage regeneration. In vitro results showed that cells adhered to the surface of the nanofibrous microparticles and migrated to the inside of the hollow structure, while remaining rounded in morphology, which is characteristic of the chondrocyte phenotype. Additionally, high production of GAGs and high levels of aggrecan and collagen II expression were detected. The capacity of the nanofibrous hollow microparticles as an injectable scaffold to fill cartilage defects was also tested in vitro using a mold with the shape of a rat femoral condyle. After 4 week of chondrocytes culture, a piece of cartilage tissue, filling the entire mold could be obtained. The engineered tissue was composed by abundant amounts of GAG and collagen II, confirmed by safranin-O and immunohistochemistry, respectively.

In a different assembly approach from engineered materials assembly, cell based therapies, such as cell sheet technology or magnetic-based approaches, are also included in hierarchical strategies for cartilage repair. Cell sheet technology using temperature-responsive culture dishes was first reported by Okano et al. (1993), and nowadays is used widely in different regenerative medicine fields. Cell sheets as hierarchical systems comprise the assembly of multiple layers of cell sheets, which then originates a 3D engineered tissue from micro to macroscales. Kushida et al. (2000) reported the production of 3D cell sheets. The engineered tissue structures had intact ECM and adhesion factors, allowing the production of a 3D construct without the use of scaffolds. Particularly for cartilage TE, Mitani et al. (2009) produced cell sheets composed by chondrocytes, with a consistent cartilaginous phenotype and adhesive properties. Sato et al. (2014) reported different studies that successfully regenerate cartilage in vitro by cell sheet technology. Additionally, in this study they solved some drawbacks reported by other authors, and confirmed the safety and efficacy of cell sheet technology for cartilage defects repair. The authors stated that a clinical study with layered 3D chondrocyte sheets is currently ongoing. Likewise cell sheet technology, a promising alternative for shaping multicellular organization is the use of magnetic forces. This approach requires individual cell magnetization through the internalization of magnetic nanomaterials. Fayol et al. (2013) reported the formation of stem cells aggregates at the millimetric scale guided by home-designed miniaturized magnetic devices, and the production of 3D cellular patterns of defined sizes and shapes. The proposed magnetic confinement was an alternative approach to the standard centrifugation for the production of cell aggregates. The in vitro differentiation of MSCs into the chondrogenic lineage and nanoparticles with a maghemite core-citrate coated were chosen as the model system. With this approach the potential use of stem cells magnetic confinement for building large cartilage tissue substitutes of defined geometry and high tissue integration potential was demonstrated.

Figure 9.4 summarizes the referred examples found in literature comprising multilayered strategies to regenerate cartilage.

All the different referred studies found in literature regarding multiphasic, multiscale, multilayered, and hierarchical strategies to regenerate cartilage tissue are summarized in Table 9.1. In each example the shape and composition of the components used, the respective assembling technique to produce 3D constructs, as well as, the cell phenotype and isolation source used to performed in vitro and/or in vivo tests were described. 


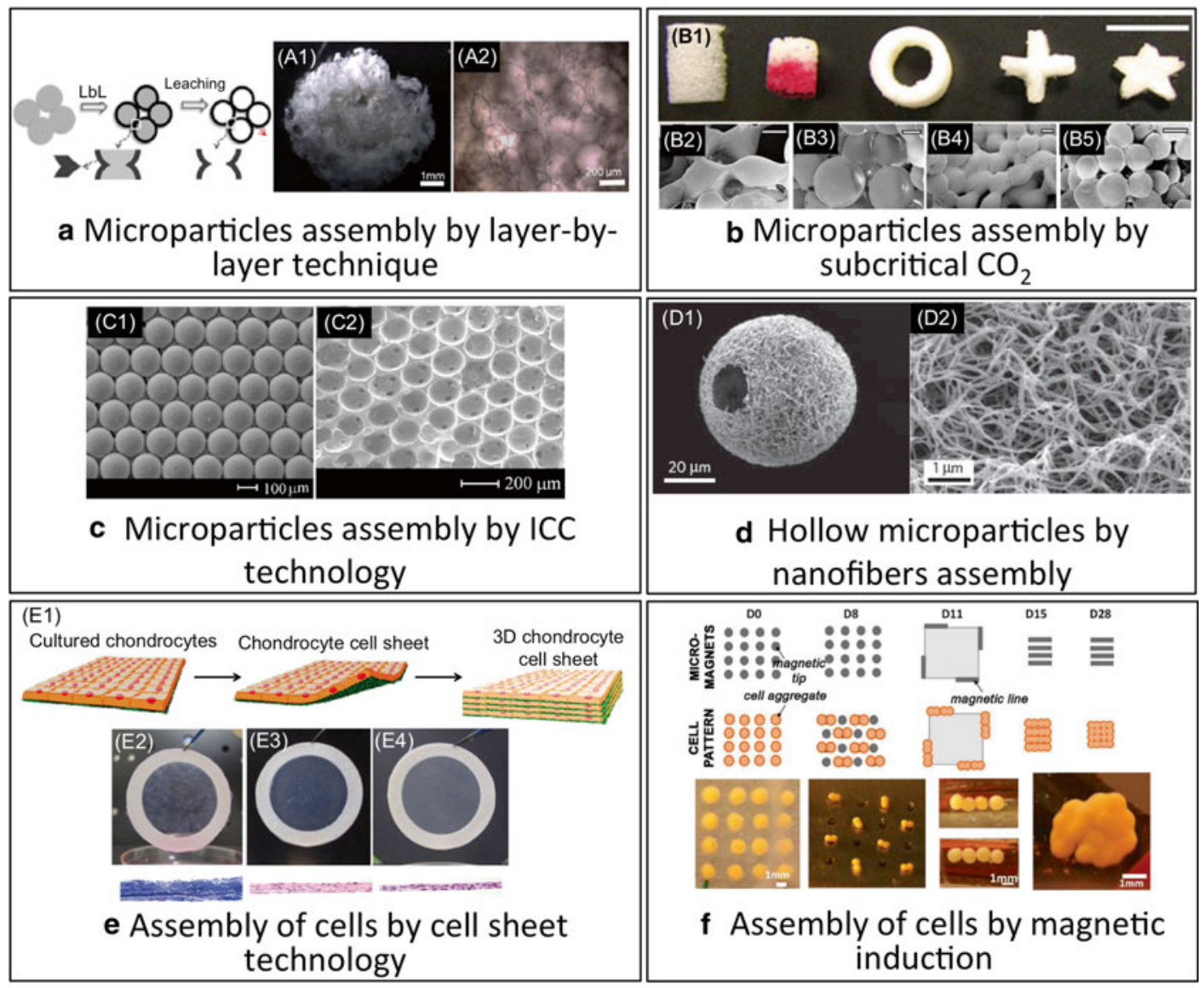

Fig.9.4 Examples of hierarchical strategies to regenerate articular cartilage. (a) Scheme of the production steps of nanostructured 3D constructs. (A1) Picture and (A2) optical microscopy of the obtained microparticles-based scaffolds fabricated combining $\mathrm{LbL}$ and leaching of free-packet paraffin spheres (Adapted from Silva et al. 2013). (b) Microparticles-based scaffolds by using subcritical carbon dioxide $\left(\mathrm{CO}_{2}\right)$ as a sintering agent to assemble poly(D,L-lactide-co-glycolide) (PLG) microparticles. (B1) Various shape-specific scaffolds produced by using rubber molds of different shapes filled with PLG microspheres (140 $\mu \mathrm{m}$ diameter) using $\mathrm{CO}_{2}$ at subcritical conditions ( 15 bar for $1 \mathrm{~h}$ at $25^{\circ} \mathrm{C}$ followed by depressurization at $0.14-0.21 \mathrm{bar}^{-1} \mathrm{~s}^{-1}$. From left to right: cylinder, bilayered cylinder, tube, plus sign, and star shapes. Scale bar is $1 \mathrm{~mm}$. (B2) Characteristic scanning electron microscopy (SEM) images of PLG scaffolds at $\mathrm{CO}_{2}$ at subcritical conditions. Sizes of the microspheres used were (B2 and B3) $240 \mu \mathrm{m},(B 4) 175 \mu \mathrm{m}$, and (B5) $140 \mu \mathrm{m}$ (Adapted from Singh et al. 2010). (c) Microparticles assembling organized in colloids with hexagonal structure by inverted colloidal crystals (ICC). SEM images of (C1) inverted colloidal crystals and (C2) heparinized ICC scaffolds in pure ethylene glycol (Adapted from Kuo and Tsai 2011). (d) Assembly of nanofibers to produce hollow microparticles without using any prefabricated template. SEM images $(D 1)$ of a nanofibrous hollow microparticle, showing the nanofibrous architecture and a hole of approximately $20 \mu \mathrm{m}$ on the microparticle shell, and (D2) showing the nanofibers, which have an average diameter of about $160 \mathrm{~nm}$ (Adapted from Liu et al. 2011). (e) Cell sheets technology for articular cartilage regeneration. (E1) Schematic representation of the production of temperature-responsive three-dimensional (3D) chondrocyte sheets. 3D cell sheets composed by chondrocytes isolated from different sources and used at different passages, namely (E2) from rabbits at passage 0 , (E3) from mini-pigs at passage 1, and (E4) from humans at passage 1 . Each cell sheet presented has its corresponding histological image showing the respective ECM deposition (bellow). Cell sheets with the same number of layers, namely three, but with chondrocytes isolated from different sources have different amounts of ECM deposition (Adapted from Sato et al. 2014). (f) Sequential magnetic fusion for the formation of a cartilage sheet. A network of magnetic tips was used to form 16 spheroid aggregates of $2.5 \times 10^{5}$ cells (day 0, D0), which were placed in contact on day 8 (D8), leading to the formation of eight doublets upon spheroid fusion. At day 11 (D11), four quadruplets were formed upon doublet fusion. Ultimately, at days 15 and 28 (D15 and D28), the four quadruplets were fused to form the final 3D structure (Adapted from Fayol et al. 2013) 


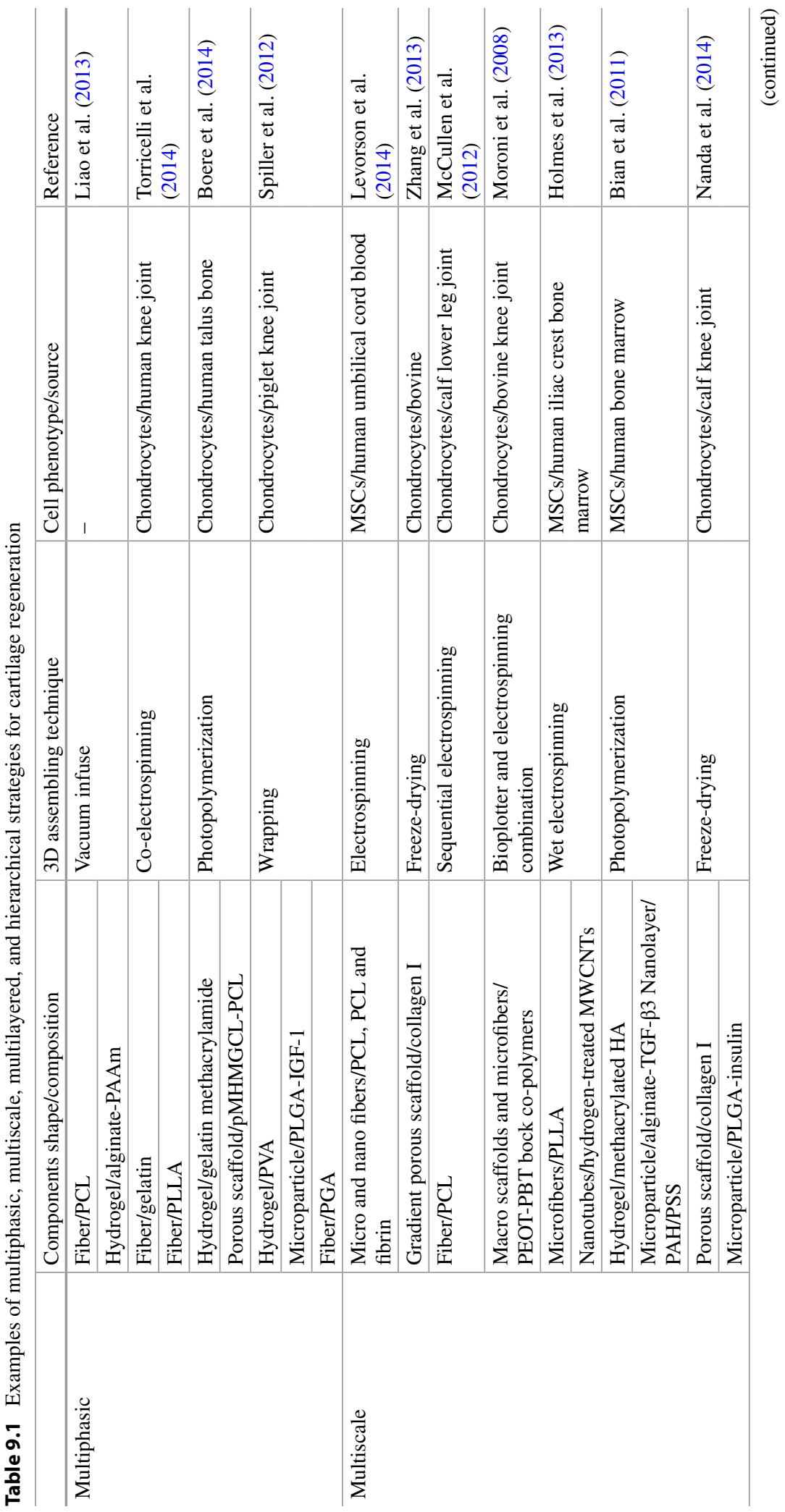




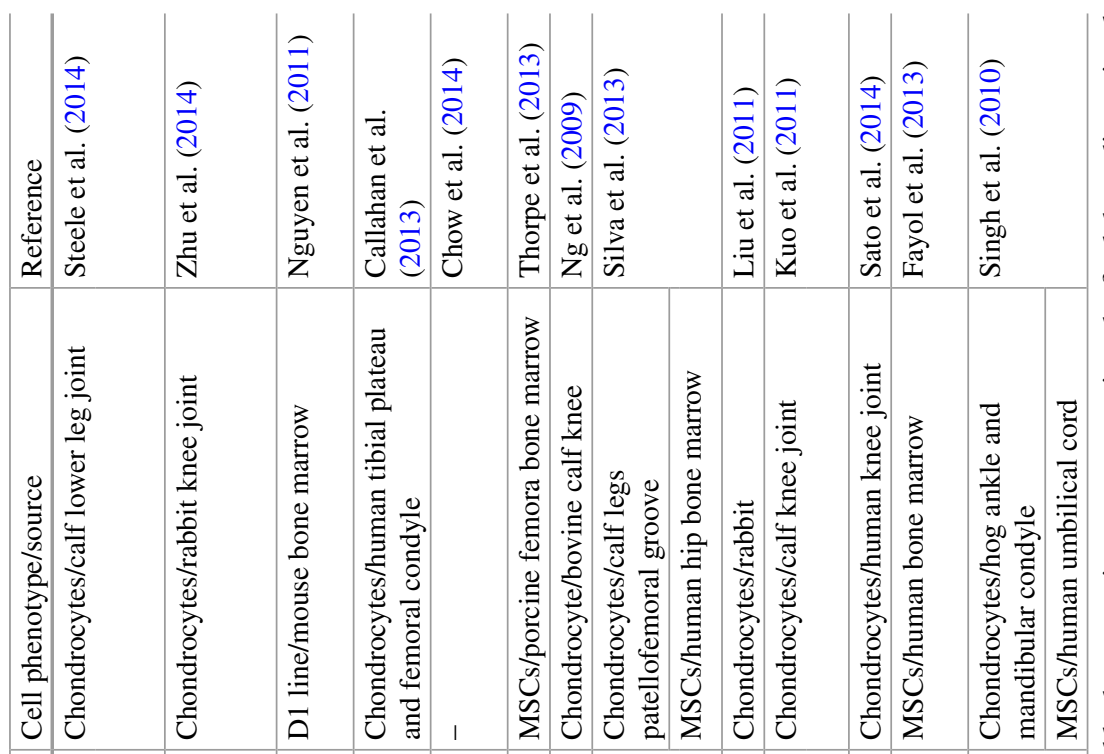

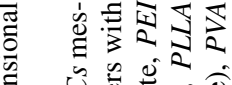

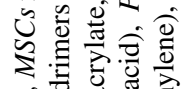

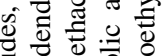
류용

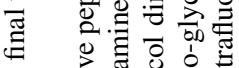
巳 :

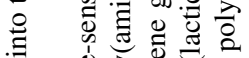

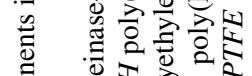
하

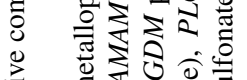
: छ च छ

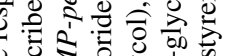
E

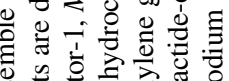

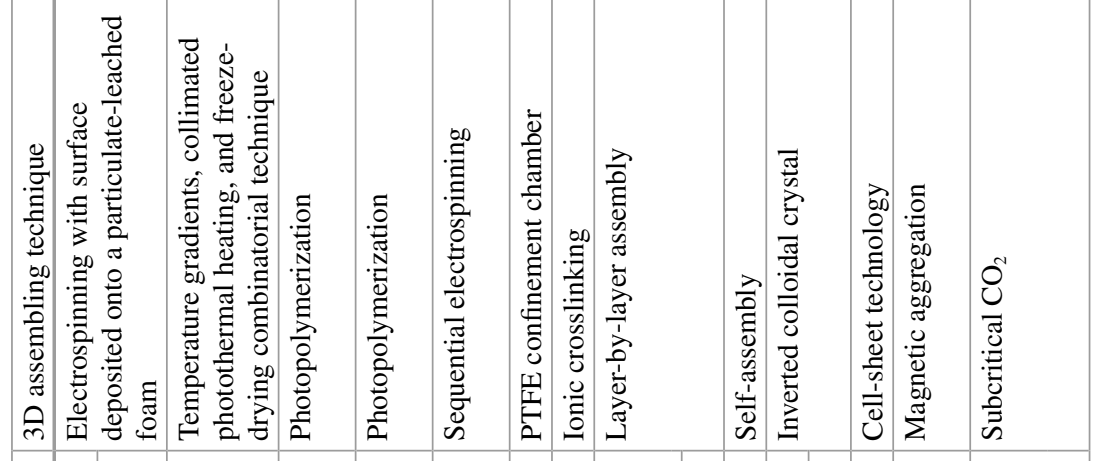
के 원

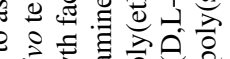

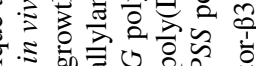

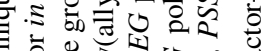
웜웡

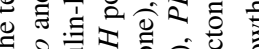

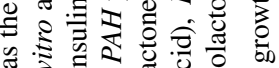

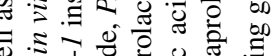

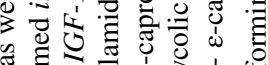

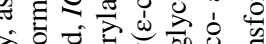

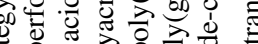

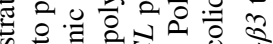

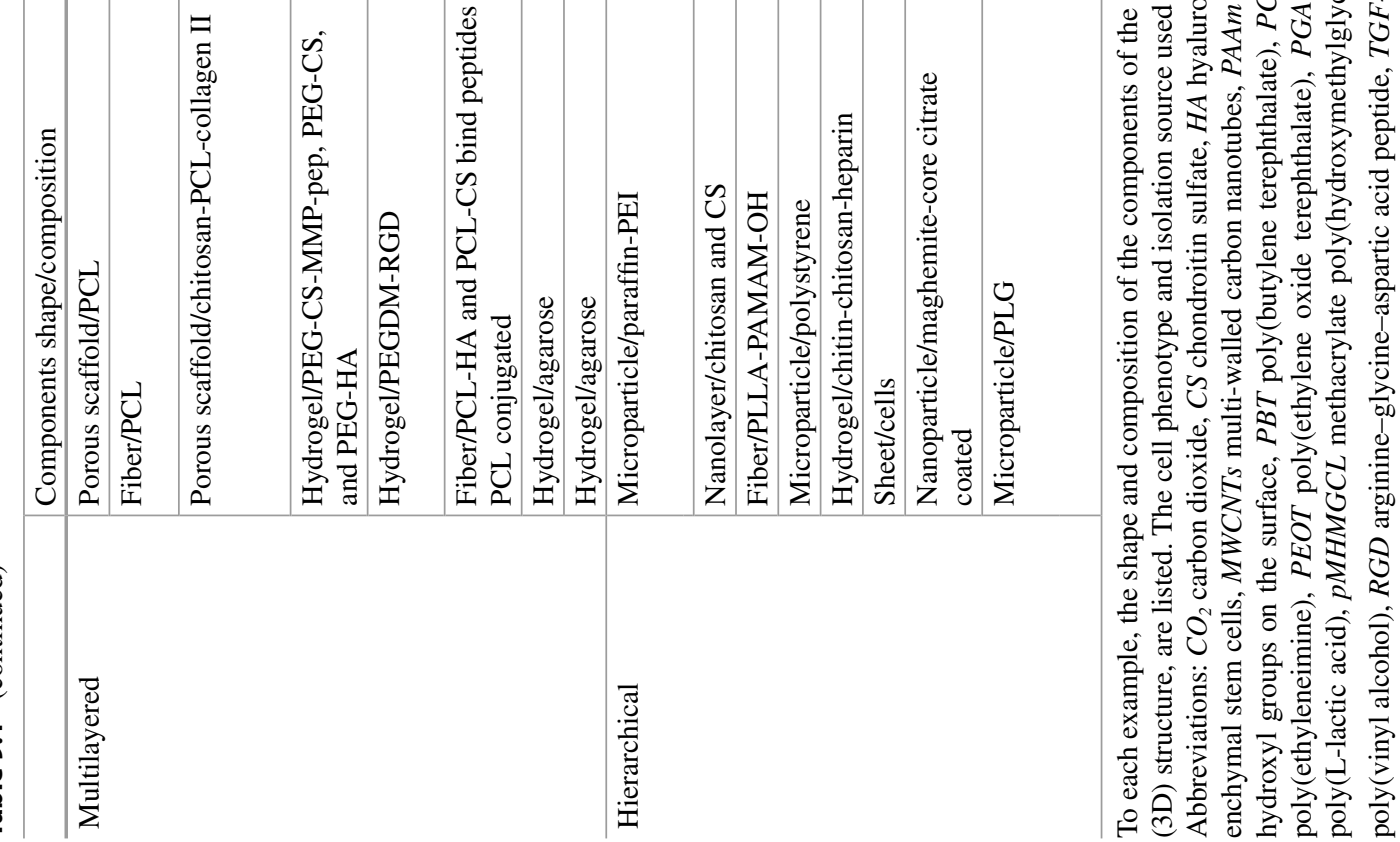

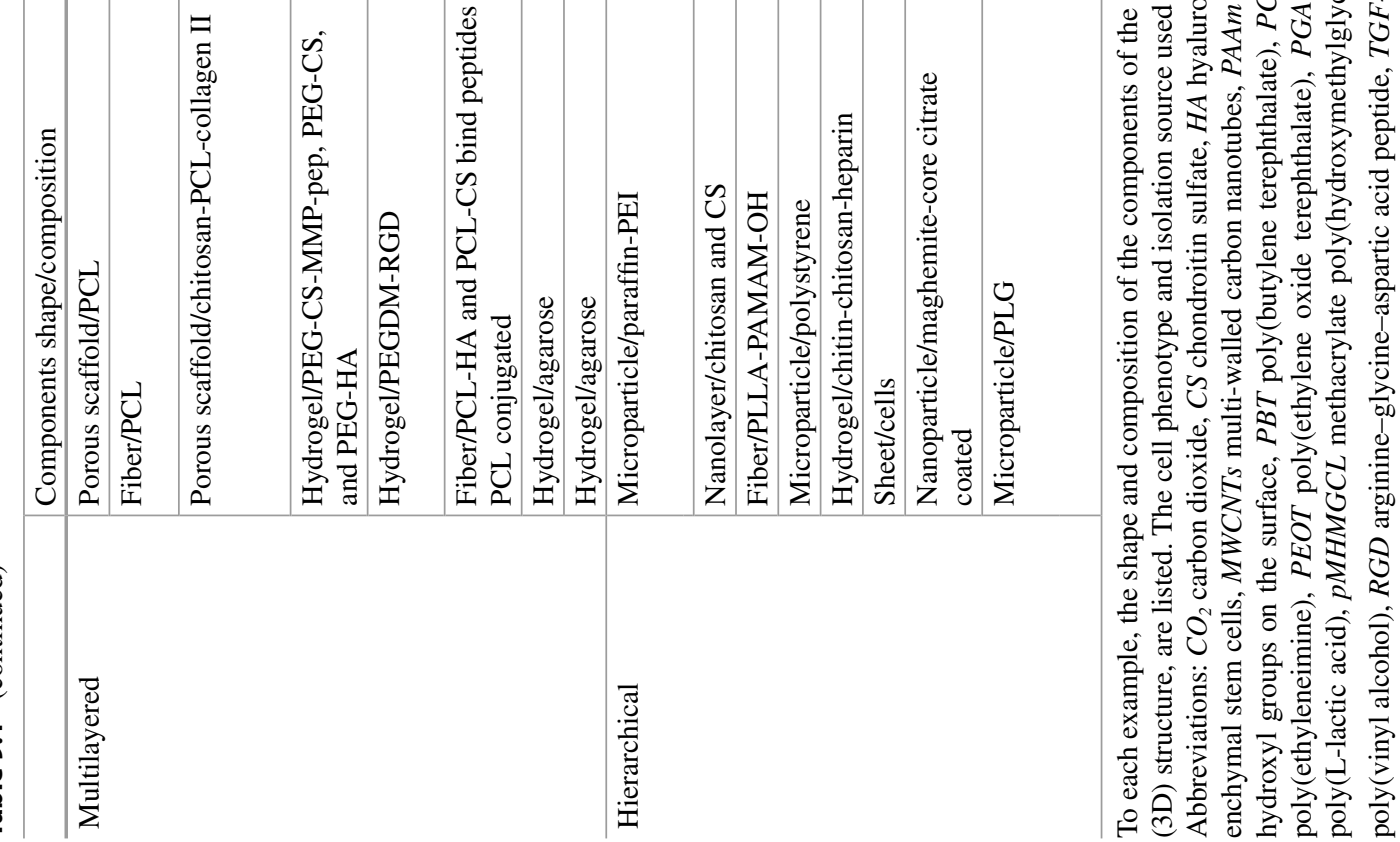




\subsection{Summary and Future Directions}

Articular cartilage is a highly organized and hierarchical tissue that provides to the bearing surface low friction and wear-resistance. The characteristic anisotropic mechanical properties of cartilage tissue are a result of depth-dependent differences in the composition of its ECM. Once damaged, cartilage tissue has a low self-repair potential. In current clinical practice, different cartilage repair techniques are being used to address tissue damage. However, their outcome remains unpredictable and long-term success is questionable. Cartilage tissue engineering has emerged as an alternative method to regenerate cartilage defects. At present, the success of cartilage TE requires a detailed understanding of the contribution of biological factors, architectural and mechanical properties, and biochemi$\mathrm{cal} /$ material composition in the regeneration of cartilage. Cartilage TE with the research and development of innovative biomaterials and research models, contributed to a more extensive understanding of cartilage biology. Particularly, either the development of biomimetic scaffolds and/or research models has contributed to the regulation and/or stimulation of different biological processes of cells, facilitating ECM deposition, alignment and functionality. Future strategies to fabricate biomaterials for cartilage TE can now have their development base on the wide range of scientific data available. Additionally, the detailed analysis of the engineered tissue using well-known techniques, such as histology, immunocytochemistry, and mechanical assays, can now be complemented with new and sophisticated characterization techniques, including nanotechnology and novel biofabrication methods, thus providing informative insights about the structure-function relationship of cartilage from its macro to nanostructure.

\section{References}

Awad HA et al (2004) Chondrogenic differentiation of adipose-derived adult stem cells in agarose, alginate, and gelatin scaffolds. Biomaterials 25(16):3211-3222
Bank RA et al (1998) Ageing and zonal variation in posttranslational modification of collagen in normal human articular cartilage: the age-related increase in non-enzymatic glycation affects biomechanical properties of cartilage. Biochem J 330(1):345-351

Bhosale AM, Richarson JB (2008) Articular cartilage: structure, injuries and review of management. Br Med Bull 87(1):77-95

Bian L et al (2011) Enhanced MSC chondrogenesis following delivery of TGF- $\beta 3$ from alginate microspheres within hyaluronic acid hydrogels in vitro and in vivo. Biomaterials 32(27):6425-6434

Boere KW et al (2014) Covalent attachment of a threedimensionally printed thermoplast to a gelatin hydrogel for mechanically enhanced cartilage constructs. Acta Biomater 10(6):2602-2611

Callahan LAS et al (2013) Maximizing phenotype constraint and extracellular matrix production in primary human chondrocytes using arginine-glycine-aspartate concentration gradient hydrogels. Acta Biomater 9(7):7420-7428

Chiang H, Jiang C-C (2009) Repair of articular cartilage defects: review and perspectives. J Formos Med Assoc 108(2):87-101

Chow LW et al (2014) Peptide-directed spatial organization of biomolecules in dynamic gradient scaffolds. Adv Healthc Mater 3(9):1381-1386

Chung C, Burdick JA (2008) Engineering cartilage tissue. Adv Drug Deliv Rev 60(2):243-262

Chung C, Beecham M, Mauck RL, Burdick JA (2009) The influence of degradation characteristics of hyaluronic acid hydrogels on in vitro neocartilage formation by mesenchymal stem cells. Biomaterials 30(26):4287-4296

Fayol D et al (2013) Use of magnetic forces to promote stem cell aggregation during differentiation, and cartilage tissue modeling. Adv Mater 25(18):2611-2616

Ge $\mathrm{Z}$ et al (2012) Functional biomaterials for cartilage regeneration. $\mathrm{J}$ Biomed Mater Res A 100(9):2526-2536

Gomoll AH, Minas T (2014) The quality of healing: articular cartilage. Wound Repair Regen 22(Suppl1):30-38

Grogan SP et al (2013) Zone-specific gene expression patterns in articular cartilage. Arthritis Rheum $65(2): 418-428$

Holmes B et al (2013) Enhanced human bone marrow mesenchymal stem cell functions in novel 3D cartilage scaffolds with hydrogen treated multi-walled carbon nanotubes. Nanotechnology 24(36):365102

Kleine TJ, Malda J, Sah RL, Hutmacher DW (2009) Tissue engineering of articular cartilage with biomimetic zones. Tissue Eng B Rev 15(2):143-157

Kuo Y-C, Tsai YT (2011) Heparin-conjugated scaffolds with pore structure of inverted colloidal crystals for cartilage regeneration. Colloids Surf B: Biointerfaces 82(2):616-623

Kushida A et al (2000) Temperature-responsive culture dishes allow nonenzymatic harvest of differentiated Madin-Darby canine kidney (MDCK) cell sheets. J Biomed Mater Res 51(2):216-223 
Levorson EJ et al (2014) Fabrication and characterization of multiscale electrospun scaffolds for cartilage regeneration. Biomed Mater 8(1):014103

Li W-J et al (2005) Multilineage differentiation of human mesenchymal stem cells in a three-dimensional nanofibrous scaffold. Biomaterials 26(25):5158-5166

Liao I-C et al (2013) Composite three-dimensional woven scaffolds with interpenetrating network hydrogels to create functional synthetic articular cartilage. Adv Funct Mater 23(47):5833-5839

Liu X, Jin X, Ma PX (2011) Nanofibrous hollow microspheres self-assembled from star-shaped polymers as injectable cell carriers for knee repair. Nat Mater 10:398-406

Malda J et al (2012) Comparative study of depthdependent characteristics of equine and human osteochondral tissue from the medial and lateral femoral condyles. Osteoarthritis Cartilage 20(10): 1147-1151

McCullen SD et al (2012) Anisotropic fibrous scaffolds for articular cartilage regeneration. Tissue Eng A 18(19-20):2073-2083

Mitani G et al (2009) The properties of bioengineered chondrocyte sheets for cartilage regeneration. BMC Biotechnol 9(17)

Moroni L et al (2008) 3D fiber-deposited electrospun integrated scaffolds enhance cartilage tissue formation. Adv Eng Mater 18(1):53-60

Mow VC, Ratcliffe A, Poole AR (1992) Cartilage and diarthrodial joints as paradigms for hierarchical materials and structures. Biomaterials 13(2):67-97

Nanda HS et al (2014) Collagen scaffolds with controlled insulin release and controlled pore structure for cartilage tissue engineering. Biomed Res Int 2014(2014): 10

Ng KW, Ateshian GA, Hung CT (2009) Zonal chondrocytes seeded in a layered agarose hydrogel create engineered cartilage with depth-dependent cellular and mechanical inhomogeneity. Tissue Eng A 15(9):2315-2324

Nguyen LH, Kudva AK, Saxena NS, Roy K (2011) Engineering articular cartilage with spatially-varying matrix composition and mechanical properties from a single stem cell population using a multi-layered hydrogel. Biomaterials 32(29):6946-6952

Okano T, Yamada N, Sakai H, Sakurai Y (1993) A novel recovery system for cultured cells using plasmatreated polystyrene dishes grafted with poly(NIsopropylacrylamide). J Biomed Mater Res A 27(10):1243-1251

Pham QP, Sharma U, Mikos AG (2006) Electrospun poly ( $\varepsilon$-caprolactone) microfiber and multilayer nanofiber/microfiber scaffolds: characterization of scaffolds and measurement of cellular infiltration. Biomacromolecules 7(10):2796-2805
Poole AR et al (2001) Composition and structure of articular cartilage: a template for tissue repair. Clin Orthop Relat Res 391(suppl):S26-S33

Sato M et al (2014) Articular cartilage regeneration using cell sheet technology. Anat Rec 297(1):36-43

Silva JM et al (2013) Nanostructured 3D constructs based on chitosan and chondroitin sulphate multilayers for cartilage tissue engineering. PLoS One 8(2):1-11

Singh M et al (2010) Microsphere-based scaffolds for cartilage tissue engineering: using subcritical $\mathrm{CO} 2$ as a sintering agent. Acta Biomater 6(1):137-143

Spiller KL et al (2012) A novel method for the direct fabrication of growth factor-loaded microspheres within porous nondegradable hydrogels: controlled release for cartilage tissue engineering. J Control Release 157(1):39-45

Steele JAM et al (2014) Combinatorial scaffold morphologies for zonal articular cartilage engineering. Acta Biomater 10(5):2065-2075

Temenoff JS, Mikos AG (2000) Review: tissue engineering for regeneration of articular cartilage. Biomaterials 21:431-440

Thorpe SD, Nagel T, Carroll SF, Kelly DJ (2013) Modulating gradients in regulatory signals within mesenchymal stem cell seeded hydrogels: a novel strategy to engineer zonal articular cartilage. PLoS One 8(4):e60764

Tian H et al (2011) Biodegradable synthetic polymers: preparation, functionalization and biomedical application. Prog Polym Sci 37(2):237-280

Torricelli P et al (2014) Co-electrospun gelatin-poly(Llactic acid) scaffolds: modulation of mechanical properties and chondrocyte response as a function of composition. Mater Sci Eng C 36(1):130-138

Tran P, Zhang L, Webster TJ (2009) Carbon nanofibers and nanotubes in regenerative medicine. Adv Drug Deliv Rev 61(12):1097-1114

Wan Y et al (2010) Biodegradability of conducting chitosan-G-polycaprolactone/polypyrrole conduits. Polym Degrad Stab 95(10):1994-2002

Williamson AK et al (2003) Tensile mechanical properties of bovine articular cartilage: variations with growth and relationships to collagen network components. J Orthop Res 21(5):872-880

Zhang Q, Lu H, Kawazoe N, Chen G (2013) Preparation of collagen porous scaffolds with a gradient pore size structure using ice particulates. Mater Lett 107:280-283

Zhu Y et al (2014) Manufacture of layered collagen/ chitosan-polycaprolactone scaffolds with biomimetic microarchitecture. Colloids Surf B: Biointerfaces 113(1):352-360 